\title{
Algorithm for constructing hedging strategies by means of Haar interpolations in the framework of the stochastic model of a one-step financial market
}

\author{
I. Tsvetkova ${ }^{1, *}$ \\ ${ }^{1}$ Don State Technical University, 1, Gagarin sq., Rostov-on-Don, 344003, Russia
}

\begin{abstract}
A stochastic model of a one-step financial market with a countable number of states is constructed. A description of the computational processes that are necessary for the development of a software package intended for calculating the components of hedging portfolios that replicate Markov-type financial obligations is presented.
\end{abstract}

\section{Introduction}

The construction of hedging strategies is one of the main problems arising in the theory of $(\mathrm{B}, \mathrm{S})$-markets. In connection with the crisis events in the world economy, there is an expansion and complication of financial markets. At the same time, it becomes necessary to create software systems that allow performing complex calculations associated with determining the fair prices of contingent claims, calculating the components of hedging portfolios. The creation of such products is based on the development of appropriate algorithms. The article deals with cases when a very large number of buyers of shares operate on the market. From a mathematical point of view, this situation is presented in the form of $(\mathrm{B}, \mathrm{S})$-a market with a countable number of states. Research methods and techniques for calculating such markets are based on the theory of Haar interpolations proposed by prof. I.V. Pavlov [1-2]. Thanks to this method, it is possible to transform incomplete non-arbitrage markets into complete non-arbitrage markets with a countable number of states, in which all calculations are carried out, necessary for the optimal behavior of investors in financial markets. For such markets, the article presents a general algorithm for calculating the components of a hedging portfolio for Markov-type contingent claims.

\footnotetext{
*Corresponding author: pilipenkoIV@mail.ru
} 


\section{Stochastic model of a one-step market with a countable number of states}

Let $\Omega$ be a countable space of elementary events, $\mathbf{F}=\left(\mathrm{F}_{0}, \mathrm{~F}_{1}\right)$ be a one-step filtration, where $F_{0}$ is trivial $\sigma$-algebra, $F_{1}$ is the $\sigma$-algebra generated by a partition $\Omega$ into countable number of atoms $B_{i}, i=1,2,3, \ldots$ Consider $\mathbf{F}$-adapted stochastic process $Z=\left(Z_{k}, \mathrm{~F}_{k}\right)_{k=0}^{1}(Z$ presents value of a share $)$. We denote the values of the process $Z$ in the following way: $Z_{0}(\Omega)=a, Z_{1}\left(B_{i}\right)=b_{i}, \quad a \in R, b_{i} \in R, i=1,2,3, \ldots, \quad(R$ is the set of all real numbers). We say that a number $b_{k}$ is of multiplicity $m_{k}, k=1,2,3 \ldots, r$, $m_{k} \leq \infty$, if in sequence $\left\{b_{i}\right\}_{i=1}^{\infty}$ it appears $m_{k}$ times. The algorithm presented in this paper is written for the case when among $\left\{b_{i}\right\}_{i=1}^{\infty} r \quad(3 \leq r<\infty)$ there are $r \quad(3 \leq r<\infty)$ different meanings. Without loss of generality, we can assume that the elements of the sequence $\left\{b_{i}\right\}_{i=1}^{\infty}$ satisfy the inequality: $b_{1}<b_{2}<\ldots<b_{r}$. Changing the numbering, we obtain the following notation:

$$
\begin{aligned}
& b_{1}=b_{r+1}=b_{2 r+1}=\ldots=b_{r(j-1)+1}=\ldots, \quad 1 \leq j<m_{1}+1 \\
& b_{k}=b_{r+k}=b_{2 r+k}=\ldots=b_{r(j-1)+k}=\ldots, \quad 1 \leq j<m_{k}+1 \\
& b_{r}=b_{2 r}=b_{3 r}=\ldots=b_{r j}=\ldots, \quad 1 \leq j<m_{r}+1 .
\end{aligned}
$$

Moreover, we assume that at least two chains of these equalities must be infinite. In sequence $\left\{B_{i}\right\}_{i=1}^{\infty}$ you can also select groups of events, at each of which the values of a random variable $Z_{1}$ coincide respectively with $\boldsymbol{b}_{1}, \boldsymbol{b}_{2}, \ldots, \boldsymbol{b}_{r}:\left\{\boldsymbol{B}_{r(j-1)+1}\right\}_{j=1}^{m_{1}} ;\left\{\boldsymbol{B}_{r(j-1)+2}\right\}_{j=1}^{m_{2}} ; \ldots ;\left\{\boldsymbol{B}_{r j}\right\}_{j=1}^{m_{r}}$.

Let us denote by:

$$
D_{k}=\bigcup_{j=1}^{m_{k}} B_{r(j-1)+k}, k=1,2, \ldots, r .
$$

Let be $\mathrm{P}=\left\{P=\left(p_{1}, p_{2}, \ldots, p_{n}, ..\right): p_{i}=P\left(B_{i}\right)>0, i=1,2,3, \ldots\right\}$ - set of nondegenerate probability measures, $P(Z, \mathbf{F}) \subset \mathrm{P}$ - set of probabilistic measures with respect to which the process $Z$ is a martingale. This set coincides with the set of solutions of the system: 


$$
\left\{\begin{array}{c}
b_{1} p_{1}+b_{2} p_{2}+\ldots+b_{n} p_{n}+\ldots=a \\
p_{1}+p_{2}+\ldots+p_{n}+\ldots=1 \\
p_{i}>0, \quad i=1,2,3, \ldots
\end{array}\right.
$$

Let us denote by:

$$
x_{k}=\sum_{j=1}^{m_{k}} p_{r(j-1)+k}, k=1,2, \ldots, r .
$$

Then system (1) can be written as:

$$
\left\{\begin{array}{c}
\sum_{k=1}^{r} b_{k} x_{k}=a \\
\sum_{k=1}^{r} x_{k}=1 \\
x_{k}>0,1 \leq k \leq r
\end{array}\right.
$$

In order for the set of solutions to system (2) to be nonempty, i.e. the original financial market was arbitrage-free, inequality must be fulfilled: $\min _{1 \leq k \leq r} b_{k}<a<\max _{1 \leq k \leq r} b_{k}$. If the market under consideration is incomplete, then for the transition to the full market we will use the Haar interpolation method, which assumes the existence of martingale measures $P \in \mathrm{P}(Z, \mathbf{F})$, satisfying a special interpolation property - the weakened noncoincidence of barycenters condition (WNBC) [3]. Many martingale process measures $Z$, satisfying WNBC, we denote $W N B C(Z)$. Sufficient conditions for existence $P \in W N B C(Z)$ received in work [4].

\section{Algorithm for financial calculations}

Step 0. Let's build a model of the initial $(1, Z)$ - market.

1) Let's define the values $r$ and multiplicity $m_{1}, m_{2}, \ldots, m_{r}$ (and at least two values must be equal to infinity).

2) Let's set the values $\mathbf{F}$ - adapted random process $Z$.

3) Let's set a limited financial obligation of the Markov type (e.g. option-call: $\left.F_{1}=\left(Z_{1}-a\right)^{+}\right)$:

$$
F_{1}=\sum_{k=1}^{r} c_{k} I_{D_{k}}
$$

Step 1. Check the financial commitment for replicability in the original market.

Let's solve the system: 


$$
\left\{\begin{array}{c}
\beta_{1}+\gamma_{1} Z_{1}\left(D_{1}\right)=F_{1}\left(D_{1}\right) \\
\beta_{1}+\gamma_{1} Z_{1}\left(D_{2}\right)=F_{1}\left(D_{2}\right) \\
\ldots \ldots \ldots \ldots \ldots \ldots \ldots \ldots \ldots \ldots \ldots \ldots \ldots \ldots \ldots \ldots \ldots \ldots \ldots \ldots \\
\beta_{1}+\gamma_{1} Z_{1}\left(D_{r}\right)=F_{1}\left(D_{r}\right) \\
\beta_{1}+\gamma_{1} Z_{0}(\Omega)=X_{0}
\end{array}\right.
$$

wherein $X_{0}=\beta_{0}+\gamma_{0} Z_{0}(\Omega)$.

If the financial liability is replicable, that is, the system in question has a solution $\pi=\left(\beta_{n}, \gamma_{n}\right)_{n=0}^{1}$, then the calculations are completed (go to step 5). If the financial liability is not replicable, then the market in question is incomplete. As it was said, the transition to the full market is carried out using the method of special Haar interpolations, which works only if there is a martingale measure that satisfies the WNBC. In what follows, we assume that the corresponding ones are satisfied.

Step 2. We work with an interpolation martingale measure.

For the model constructed at step 0 , we find a martingale measure that satisfies the WNBC. We will get the corresponding values of the sums:

$$
x_{k}=\sum_{j=1}^{m_{k}} p_{r(j-1)+k}, k=1,2, \ldots, r .
$$

2) Calculating the price of a financial liability:

$$
C\left(F_{1}\right)=\sum_{k=1}^{r} c_{k} x_{k} .
$$

Step 3. Build a special Haar interpolation of the original market.

Due to the fact that we are considering financial markets with a countable number of states, we will use quantile hedging. To determine the computational horizon $N$ the following actions will be required:

- $\quad$ set the accuracy $\varepsilon$;

- a typical scheme of special Haar filtering is a scheme when, when passing from the moment of time $n$ to the moment $n+1, n=1,2,3, \ldots$ only one atom is crushed, and the one that was obtained as a result of crushing in the previous step; given the fact that the order of occurrence of events $B_{i}, i=1,2, \ldots$ at intermediate times is not defined, so we represent it as a vector $\delta=\left(\delta^{(1)}, \delta^{(2)}, \ldots\right)$. Vector coordinates $\delta$ dependent random variables and will be determined using a random vector generator based on geometric distribution [5];

- for each random event $B_{\delta^{(n)}}, n=1,2, \ldots, N$ using a special algorithm, we find the values of the components of the martingale measure obtained at step 2 ; 
- check the inequality: $\sum_{i=1}^{N} p_{\delta^{(i)}}>1-\varepsilon$. If the inequality is true, then $N$ computational horizon for the interpolating market; filtration $\mathbf{H}$, interpolating filtering $\mathbf{F}$, represents: $\mathrm{H}_{0}=\mathrm{F}_{0}, \mathrm{H}_{1}=\sigma\left\{B_{\delta^{(1)}}\right\}, \ldots, \mathrm{H}_{N}=\sigma\left\{B_{\delta^{(1)}}, B_{\delta^{(2)}}, \ldots, B_{\delta^{(N)}}\right\}$.

2) Solving the Dirichlet martingale problem with a boundary random variable $Z_{1}$, we obtain the values of the random process $\left(Y_{k}\right)_{k=0}^{N}$, interpolating process $Z$ :

$$
Y_{n}=\sum_{k=1}^{n} b_{\delta^{(k)}} I_{B_{\delta^{(k)}}}+\frac{a-\sum_{k=1}^{n} b_{\delta^{(k)}} p_{\delta^{((k))}}}{1-\sum_{k=1}^{n} p_{\delta^{(k)}}} I_{\bar{B}_{n, \delta}(n)}, \quad n=1,2, \ldots, N,
$$

where $\bar{B}_{n, \delta^{(n)}}=\overline{B_{\delta^{(1)}}+B_{\delta^{(2)}}+\ldots+B_{\delta^{(n)}}}, n=1,2, \ldots, N$.

Step 4. Calculate the components of the self-financing portfolio and its total capital.

Let $\pi=\left(\beta_{n}, \gamma_{n}\right)_{n=0}^{\infty}$ - self-financing portfolio, where $\left(\beta_{n}\right)_{n=0}^{\infty}$ и $\left(\gamma_{n}\right)_{n=0}^{\infty}$ — predictable with respect to $\mathbf{H}$ sequences [6]:

$$
\begin{array}{ll}
\gamma_{n}=\sum_{k=1}^{n-1} \gamma_{n}^{\delta^{(k)}} I_{B_{\delta}(k)}+\gamma_{n}^{\delta^{(n)}} I_{\bar{B}_{n-1, \delta}(n-1)}, & n=1,2,3, \ldots \\
\beta_{n}=\sum_{k=1}^{n-1} \beta_{n}^{\delta^{(k)}} I_{B_{\delta^{(k)}}}+\beta_{n}^{\delta^{(n)}} I_{\bar{B}_{n-1, \delta}(n-1)}, & n=1,2,3, \ldots
\end{array}
$$

For convenience, we will assume that: $\gamma_{n}^{\delta^{(k)}}=0, \quad k=1,2,3, \ldots, n-1, n \geq 2$.

Known [7-8]:

$$
\begin{gathered}
\gamma_{n}^{\delta^{(n)}}=\frac{c_{\delta^{(n)}}-C\left(F_{1}\right)+\sum_{k=1}^{n-1} c_{\delta^{(k)}} p_{\delta^{(k)}}}{b_{\delta^{(n)}}-a+\sum_{k=1}^{n-1} b_{\delta^{(k)}} p_{\delta^{(k)}}}, n=1,2,3, \ldots \\
\beta_{n}^{\delta^{(k)}}=c^{\delta^{(k)}}, k=1,2,3, \ldots, n-1, n \geq 2, \\
\beta_{n}^{\delta^{(n)}}=c_{\delta^{(n)}}-\gamma_{n}^{\delta^{(n)}}, n=1,2,3, \ldots
\end{gathered}
$$

Total portfolio capital $\pi=\left(\beta_{n}, \gamma_{n}\right)_{n=0}^{\infty}$ calculated by the formula: 


$$
X_{n}=E^{P}\left[f \mid H_{n}\right]=\sum_{k=1}^{n} c_{\delta^{(k)}} I_{B_{\delta^{(k)}}}+\frac{\sum_{k=n+1}^{\infty} c_{\delta^{(k)}} p_{\delta^{(k)}}}{\sum_{k=n+1}^{\infty} p_{\delta^{(k)}}} I_{\bar{B}_{n, \delta}^{(n)}}, \quad n=1,2,3, \ldots
$$

Because $\beta_{n}=\beta_{n}^{n}=\beta_{n}^{n+1}=\ldots, \gamma_{n}=\gamma_{n}^{n}=\gamma_{n}^{n+1}=\ldots, \quad \forall n=0,1,2, \ldots$

$$
\lim _{n \rightarrow \infty} X_{n}=F_{1} \quad P \text { - a.s. [9], }
$$

then we get the full market, interpolating the original one.

Step 5. End of the algorithm.

\section{Conclusion}

The presented algorithm makes it possible to apply the method of special Haar interpolations to real calculations in arbitrage-free financial markets. It can be used as a basis for the corresponding software package, due to which the choice of the optimal strategies of investors in the financial markets will be greatly simplified.

\section{Acknowledgements}

This research is supported by the RFBR (project 19-01-00451)

\section{References}

1. Pavlov I V 2002 Russian Math Surveys 57(3) 581-583

2. Pavlov I V 2002 Isvestiya VUZov, Severo-Kavkaz, Region, Estestvenn Nauki 3 16-24

3. Danekyants A G 2004 Obozrenie Prikl Promyshl Mat 11(3) 506-508

4. Pavlov I V 2016 Theory ProbabAppl 61(1) 173-181

5. Vadzinsky R N 2001 Handbook of probability distributions (Nauka) p 296

6. Shiryaev A N 1998 Financial Mathematics. Volume 1 Facts Models (M: Fazis) p 512

7. Tsvetkova I V 2013 Electronic Scientific Edition "Naukovedenie: Internet magazine» 3(16)

8. Shamraeva V V 2014 Vestnik Moscow University Named S U Vitte Series 1: Economics and management 1

9. Shiryaev A N 2002 Proceedings of the Steklov Institute of Mathematics 237 12-56 